Received Date : 27-Jul-2016

Revised Date : 01-Oct-2016

Accepted Date : 05-Nov-2016

Article type : Original Article

\title{
Immunohistochemical expression of podoplanin (D2-40), lymphangiogenesis and neoangiogenesis in tooth germ, ameloblastomas and ameloblastic carcinomas
}

Running title: podoplanin expression in ameloblastomas

Key Words: ameloblastoma, ameloblastic carcinoma, podoplanin, lymphangiogenesis, microvascular density.

Celeste Sánchez-Romero ${ }_{1}$, DDS, Ronell Bologna-Molina 2 , DDS, PhD, Adalberto Mosqueda-Taylor ${ }_{3}$ DDS, MSc, Oslei Paes de Almeida ${ }_{1}$ DDS, PhD.

1.- Oral Pathology Section, Department of Oral Diagnosis, Piracicaba Dental School, University of Campinas (UNICAMP).

2.- Molecular Pathology Area, School of Dentistry, Universidad de La República (UDELAR), Montevideo, Uruguay.

3.- Health Care Department, Universidad Autónoma Metropolitana Xochimilco, Mexico City.

This article has been accepted for publication and undergone full peer review but has not been through the copyediting, typesetting, pagination and proofreading process, which may lead to differences between this version and the Version of Record. Please cite this article as doi: $10.1111 /$ jop. 12524

This article is protected by copyright. All rights reserved. 
Corresponding author: Celeste Sánchez Romero. Oral Pathology Section, Department of Oral Diagnosis, Piracicaba Dental School, University of Campinas (UNICAMP). Av. Limeira 901, P.O. Box. 52, 1314-903, Piracicaba, São Paulo, Brazil. E-mail: csr_90@hotmail.com Fax: +55 1921065218.

\section{ABSTRACT}

BACKGROUND: Ameloblastoma is a benign but locally aggressive odontogenic tumor, while ameloblastic carcinoma is its malignant counterpart. Angiogenesis and lymphangiogenesis in malignancies have been correlated with higher aggressiveness and poor prognosis, as well as greater expression of podoplanin by tumoral cells.

METHODS: Immunohistochemical expression of podoplanin, CD34 and CD105 (endoglin) was evaluated in 53 ameloblastomas and 3 ameloblastic carcinomas; additionally, immunohistochemistry for podoplanin was also performed in 10 tooth germs. Microvessel density of blood and lymphatic vessels was calculated and compared between ameloblastomas and ameloblastic carcinomas. Immunoexpression of podoplanin by ameloblastic cells was evaluated in tooth germs, ameloblastomas and ameloblastic carcinomas.

RESULTS: Podoplanin was similarly expressed by odontogenic epithelial cells of tooth germs and ameloblastomas, while its expression was lower in ameloblastic carcinomas. There was not difference in microvessel density assessed by CD34 between ameloblastomas and ameloblastic carcinomas; nevertheless, the latter presented higher amounts of lymphatic and new formed blood vessels.

CONCLUSIONS: Results suggest that podoplanin does not seem to be involved in invasion mechanisms of ameloblastic carcinomas, as its expression was decreased in the malignant tumoral cells. On the other hand, the increased lymphatic microvessel density and neoangiogenesis found in ameloblastic carcinomas could be related with its aggressiveness and potential for metastasis.

This article is protected by copyright. All rights reserved. 


\section{INTRODUCTION:}

Ameloblastoma (AM) is a benign but aggressive odontogenic tumor with high potential for local invasion and recurrence, particularly the solid subtype. Therefore, AM, which is an important cause of large surgeries in the maxillofacial region (1,2). Ameloblastic carcinoma $(A C)$ is the malignant counterpart of $A M$, with less than 120 cases reported in the English-language literature (3). CD34 is an immunomarker of blood vessel endothelial cells and CD105 (endoglin) is for neoformed vessels, while podoplanin is considered as a reliable immunomarker of lymphatic endothelium. These markers are commonly used to evaluate angiogenesis and lymphangiogenesis in tumors $(4,5)$.

The high expression of podoplanin in the basal layer of the oral epithelium and in tooth germ (TG) epithelium, as well as in several squamous cell carcinomas and central nervous system tumors, may be possibly related to ectodermal cell proliferation (6). Podoplanin also seems to be involved in odontoblasts growth and with the extension of odontoblastic fibers, which is mediated by cytoskeleton rearrangements. Thus, reduction of odontoblastic expression of podoplanin is observed with the completion of tooth formation (7).

Podoplanin can be expressed both in specific cell types of normal tissues as well as in tumoral cells of benign and malignant neoplasms, and it has been correlated with aggressiveness, tumor spread, metastasis and poor prognosis (8).

The aims of this study are to describe and quantify angiogenesis and lymphangiogenesis in $A M$ and $A C$, and to compare the expression of podoplanin in ameloblastic cells of TG, ameloblastomas and ameloblastic carcinomas, to better understand if these parameters might be associated with the aggressiveness of these tumors.

This article is protected by copyright. All rights reserved. 


\section{MATERIALS AND METHODS}

A total of 10 human TG (one in bud, six in cap and three in bell stages of development), $53 \mathrm{AM}$, consisting of 38 solid ameloblastomas (SA), 24 of which were plexiform (63.2\%) and 14 follicular (36.8\%); and there were 15 unicystic ameloblastomas (UA), 4 of which were (26.6\%) intraluminal, 4 (26.6\%) mural, and 7 (46.8\%) simple/luminal type;-and 3 AC were retrieved from the files of the Laboratory of Oral Pathology at the Universidad Autónoma Metropolitana-Xochimilco (Mexico), Dental School of Piracicaba (Brazil) and the Histology Department at the Universidad de la República (Uruguay). Using H\&E stained sections, all $A M$ and $A C$ were reviewed according to the more recent Histological Classification of Head and Neck Tumors of the World Health Organization (9). The current study was approved by the Ethical Committee of Piracicaba Dental School, State University of Campinas (register number 133/2014).

Immunohistochemical reactions were performed in 3- $\mu \mathrm{m}$ sections of formalin-fixed, paraffin-embedded tissues that were dewaxed with xylene and then hydrated in an ethanol series. Antigen retrieval was done by immersing the sections for 3 minutes in citrate buffer solution ( $\mathrm{pH} \mathrm{6.0)}$ in a pressure cooker for podoplanin and CD34 reactions; while for CD105, enzymatic digestion was applied for 5 minutes using Proteinase $K$ (DAKO, Cytomation, Carpinteria, CA, USA. Ready-to-use). Endogenous peroxidase activity was blocked using $10 \%$ hydrogen peroxide in 5 baths, each of 5 minutes. Subsequently, preparations were washed in phosphate buffer solution buffer ( $\mathrm{pH} 7.4)$, and incubated overnight with the primary antibody Anti-Human Podoplanin (Dako Cytomation, Carpinteria, CA, USA, clone D2-40, diluted 1:100); anti CD34 (Dako Cytomation, Carpinteria, CA, USA, clone QBEnd-10, diluted 1:50) and anti CD105 (Endoglin) (Dako Cytomation, Carpinteria, CA, USA, clone SN6h, diluted 1:30). All slides were then exposed to avidin-biotin complex and horseradish peroxidase reagents (LSAB Kit; Dako Cytomation, Carpinteria, CA, USA) and diaminobenzidine tetrahydrochloride (DAB; Sigma-Aldrich, St Louis, MO, USA). Finally, the tissue sections were counterstained with Carazzi hematoxylin. Kaposi sarcoma was used as positive control for podoplanin and pyogenic granuloma for CD34 and CD105; while the negative control was obtained by omitting the specific primary antibody.

This article is protected by copyright. All rights reserved. 
The expression of podoplanin in the ameloblastic cells was evaluated in the whole section of each case, performing a modification of the method of Yuan et al. (10), which takes into consideration three main features: the percentage of positive tumoral area (from $0 \%$ to $100 \%$ ), intensity of staining (weak, moderate and strong, based on the optical density of stain), and the patterns of expression within tumoral nests; however, differently from the above mentioned authors, we did not use scoring, describing separately these three aspects. Three patterns of expression within tumoral nests were considered: 1) Intense staining at the peripheral/basal layer of islands (ameloblasticlike cells) and weak or negative at central cells (stellated reticulum-like cells); 2) higher expression at the central/suprabasal cells (stellated reticulum-like cells) than in peripheral/ameloblastic cells; and 3) full staining of islands, with similar intensity in center and peripheral cells.

\section{Lymphatic microvascular density (LMVD)}

Due to the low quantity of lymphatic vessels (LV), the LMVD was obtained manually, based in the method initially described by Weidner (11), and used in several studies to determine microvessel density $(12,13)$. Briefly, at low magnification $(10 x)$ three "hotspots" with high number of podoplanin positive LV are localized. Subsequently, counting was performed in each area at a high power magnification field (40x). LMVD was expressed as the number of LV per square millimeter, and it was obtained by dividing the total number of LV by the total area analyzed in $\mathrm{mm}^{2}$ for each case, i.e $0.456 \mathrm{~mm}^{2}$.

Digital analysis for Microvessel Density (MVD)

The MVD of blood vessels stained with CD34 and CD105 was obtained by digital analysis. The slides were scanned into high-resolution images using the Aperio Scanscope CS Slide Scanner (Aperio Technologies Inc, Vista, CA, USA). All digital images obtained in .svs format were visualized with the ImageScope software (Aperio 
Technologies Inc). Microvessel Analysis v1 algorithm (Aperio Technologies Inc) was used to quantitate the number of vessels by CD34 and CD105 reactivity. The values of MVD were expressed as the number of vessels per unit area $\left(\mu \mathrm{m}^{2}\right)$ (Fig. 1).

Statistical analysis was performed for LMVD and MVD separately, using testing frequencies for qualitative variables, whereas for the quantitative data, comparative analyses were performed using the $x^{2}$ test, Student $t$ test, and ANOVA test. We have added nonparametric statistics for comparison of the 3 groups using the Kruskal-Wallis test. The results were considered significant when $p \leq .05$.

\section{RESULTS}

All TGs expressed podoplanin in the epithelial elements of the enamel organ. The percentage of positive area of odontogenic epithelial tissue varied from $50 \%$ to $100 \%$, with a mean of $79 \%$. Localization of staining in the TG cells varied slightly throughout the maturation stage. In the bud stage, podoplanin showed intense membrane/cytoplasmic expression in both peripheral ameloblastic and central cells that would lead to stellate reticulum, and this expression was also seen in continuity with the basal layer of the oral epithelium (Fig. 2 A). In cap stage, podoplanin was intensely positive in the cytoplasm of cells of the inner and outer enamel epithelium, with focally negative areas at the cervical loop, stratum intermedium and stellated reticulum (Fig. 2 B). At the bell stage, the pre-secretory and secretory ameloblasts had strong membrane positivity and clear cytoplasm, with the recently differentiated odontoblasts and adjacent dental papilla also showing cytoplasmic and membrane positivity respectively (Fig. $2 \mathrm{C}$ ).

All cases of AM presented membrane and cytoplasmic positivity for podoplanin: in 8 (14\%) cases staining was weak, in $13(22.8 \%)$ moderate and in $36(63.2 \%)$ strong. Percentage of positive tumoral area ranged from $10 \%$ to $100 \%$ with a mean of $84 \%$. In $82.4 \%$ of $\mathrm{AM}$ podoplanin was expressed strongly at the peripheral/basal ameloblastic cells, being weak/negative in the central/suprabasal cells that resemble the stellated reticulum (Fig. 2 D); while $17.6 \%$ of the cases, stained with similar intensity in both

This article is protected by copyright. All rights reserved. 
center and peripheral cells (Fig. 2 E). There were not any AM positive predominantly at the center of the islands and negative at the periphery. In most cases, focal negative areas were found.

Thirty-three out of 38 cases of SA (86.85\%) presented podoplanin expression predominantly at the peripheral/ameloblastic layer (Fig. 2 D), while $5(13.15 \%)$ showed similar staining in peripheral and central cells of tumor islands (Fig. $2 \mathrm{E}$ ). Comparing the expression of podoplanin between $S A$ and $U A$, we observed a similar pattern of expression; however, the percentage of positive tumoral area was lower in SA (62\% of positivity) than in UA ( $88 \%$ of positivity). Twelve of 15 cases of UA $(80 \%)$, showed podoplanin positivity restricted to the basal layer, while in 3 cases $(20 \%)$ the whole thickness of the epithelial cystic lining (basal and suprabasal layers) was positive. Similar to SA, most cases of UA had intense staining.

The mean LMVD of the 53 cases of AM was 18.7 (20.2 for SA and 13.8 for UA). In 12 (24.5\%) cases of AM, LV were not found (7 SA representing $18.4 \%$ of SA and 5 UA representing $33.3 \%$ of $U A$ ). It was difficult to determine tumoral LV hotspots in AM because of the scarce presence of LV (Fig. 2 F).

The 3 cases of $A C$ expressed podoplanin ranging from $10 \%$ to $50 \%$, with a mean of $27 \%$ of positive tumor area. In contrast to $\mathrm{AM}$, two $\mathrm{AC}(66.6 \%)$ stained predominantly at the center of the islands, with weak to strong intensity (Fig. $3 \mathrm{~A}$ and B), and one case had only $10 \%$ of weak positivity, mainly in cell clusters within islands. More frequently, extensive negative areas were observed in AC than in AM.

The mean LMVD of the 3 AC was 73.09. In contrast to AM, LV hotspots adjacent to tumoral cells were commonly observed (Fig. $3 \mathrm{C}, \mathrm{D}$ ). In AC the LMVD index was statically higher than in AM (Mann Whitney Test, $p=0.000$ ), while it was similar when SA and UA were compared (Fig. 4). In TG, lymphatic vessels were not present. As positive control, lymphatic vessels were observed adjacent to the oral epithelium.

In all cases of AM and AC, immunoreactivity for CD34 and CD105 was detected in the cytoplasm of endothelial cells of blood vessels in the peritumoral and intratumoral stroma (Fig. 5). In most follicular SA and UA, positive vessels had a peritumoral

This article is protected by copyright. All rights reserved. 
disposition (Fig. 5 A, B); while in most cases of plexiform SA and AC the vessels were frequently located in intratumoral niches (Fig. 5 C-F).

Mean values of MVD in AM and AC are shown in Figure 6. MVD assessed by CD34 (MVD-CD34) was similar between SA and UA, and between AM and CA; however, MVD evaluated with CD105 (MVD-CD105) showed a tendency to be higher in SA than in UA, and it was significantly higher in AC than in AM (Fig. 6).

There were not significant differences in the expression of podoplanin, LMVD and MVD between histologic variants of UA (mural, intraluminal and luminal), nor between subtypes of SA (plexiform and follicular).

Blood vessels were not quantified in TG, and because of the reduced size of the area in which they could be found, consequently it could not be applied the same methodology of vessel counting used for $A M$ and $A C$ (five areas of $600,000 \mu \mathrm{m} 2$ analyzed in each case). Also, we considered that the ectomesenchymal tissue that surrounds TG (dental papilla and dental follicle) is different from the fibrous connective tissue that conforms the stroma of $A M$ and $A C$.

\section{DISCUSSION}

Our results confirm that podoplanin is strongly expressed in TG, with slight pattern variations along maturation stages, according to previous studies in mouse and human TG (7). These results indicate that podoplanin plays a role in tooth development by cytoskeleton rearrangements of epithelial odontogenic cells and odontoblasts $(6,14)$. All AM also expressed podoplanin strongly, confirming recent studies $(15,16)$. There are no previous reports of podoplanin in $A C$, but our results show that expression is decreased on this malignancy in relation to AM. In neoplastic tissues podoplanin has been related to higher aggressiveness, but this was not confirmed for $S A$ and $A C$ in relation to $U A$ which is well accepted to be a less aggressive ameloblastic tumor (14, 16). As cited, TG used as normal control was strongly positive for podoplanin, and UA showed a similar or slightly higher expression than SA. In fact, AC was the group with

This article is protected by copyright. All rights reserved. 
the lowest expression, suggesting that podoplanin does not seem to play an important role related to the aggressiveness of this malignancy. These results should be carefully considered as we studied only three cases of $A C$, which is a very rare malignant odontogenic tumor.

Expression of podoplanin in AM and other odontogenic tumors and cystic odontogenic lesions has been described most frequently as positive at the peripheral/basal cells and negative or slightly positive at central/suprabasal cells $(14,16)$; however, $15 \%$ of AM (8 cases) in our series presented strong positivity in both peripheral and central cells. Tjioe et al. (18) have related the peripheral/basal pattern of positivity with cell activity, since less active cells such as non-secreting ameloblasts from odontoma are negative or slightly positive. Also, expression of podoplanin in ameloblastic cells adjacent to tumoral stroma, may be induced by stromal factors as epidermal growth factor (EGF), fibroblast growth factor 2 (FGF-2) and transforming growth factor-beta (TGF- $\beta$ ) (15). The role of podoplanin in developmental, reactive and neoplastic odontogenic epithelium is not yet well established, but it could be related to cytoskeletal reorganization in physiologic and pathologic processes as for example TG morphodifferentiation, local expansion of odontogenic cystic lesions and invasion/migration in some odontogenic tumors such as AM $(15,14)$.

This is the first study that describes, quantitates and compares the LV in TG, AM and AC. In TG, LV were absent, AM presented scarce LV that frequently were not closely associated with the ameloblastic epithelium, while in AC the LV were mostly adjacent to the tumoral cells. Recently, Sousa-Neto et al. (2012) (19) found higher number of LV in keratocystic odontogenic tumor (KCOT) as compared to radicular cysts and pericoronal follicles, and the localization of LV in KCOT were distant from the epithelium, likewise to our observations in AM. AC presented a significantly higher LMVD than AM, and this could play a role in favoring metastases by lymphatic tumoral dissemination, since around a third of cases of $A C$ results in tumor related death or pulmonary metastasis (9).

This article is protected by copyright. All rights reserved. 
Watanabe et al (2013) (20) found in squamous cell carcinomas of lip and oral cavity, a decrease of LMVD with tumor progression using podoplanin as a marker, possibly due to damage of LV by tumoral cells. Conversely, in our study, we found a significantly higher quantity of $L V$ in $A C$ than in $A M$ and TG. To confirm these findings in $A C$ it would be interesting to study additional cases of this very rare neoplasm and compare the LMVD across the tumoral progression.

CD34 is consistently positive for vascular endothelium while CD105 is highly expressed in endothelium of neoformed or angiogenic active vessels, but minimally expressed in preexistent and quiescent vessels $(21,22)$. Therefore, they are routinely used to quantitate total and neoformed blood vessels, respectively (5). It is controversial if MVD is similar or not in SA and UA $(2,23)$, and our results did not show significant differences when comparing MVD-CD34 in SA and UA.

Nevertheless, MVD-CD105 immunoexpression showed a tendency to be higher in SA than UA $(p=0.062)$. A higher MVD was described in recurrent and malignant ameloblastomas than in primary ameloblastomas $(2,23,24)$, but we found that $A M$ and AC did not present differences in MVD-CD34. However, MVD-CD105 was significantly higher in $A C$ than in $A M$, suggesting that $A C$ has a greater angiogenic potential.

Tumor growth depends of blood supply for nutrients and oxygen needs, thus higher neoangiogenesis and LMVD in AC could be related with expression of hypoxia-inducible factor-1 $\alpha$ (HIF-1 $\alpha$ ), as we showed in a previous study (25), as this transcriptor factor of VEGF and VEGF-C promotes angiogenesis and lymphangiogenesis $(14,26)$. In fact, MVD in AM was related to the expression of VEGFs by the odontogenic tumoral cells (4).

In summary, this is the first study that compares podoplanin expression in ameloblastic cells of TG, AM and AC, and also to quantitate LMVD and to compare the total amount of blood vessels versus neoformed vessels in $A M$ and $A C$. AC showed higher peritumoral lymphatic vascularization which could be related with its metastatic potential. $T G$ and $A M$ had similar expression of podoplanin, while in $A C$ it was significantly decreased, suggesting that podoplanin may not be related with the

This article is protected by copyright. All rights reserved. 
aggressiveness of this malignant odontogenic tumor. Also higher neoangiogenesis was present in the more aggressive $S A$ and $A C$, than in UA. Future studies with larger samples of AC may contribute to a better understanding of the role of podoplanin, lymphangiogenesis and neoangiogenesis in the pathogenesis and biologic behavior of malignant odontogenic tumors.

\section{Acknowledgments}

We greatly appreciate the assistance of Prof. Alvaro Maglia and Prof. Gabriel Tapia (Histology Department, Universidad de la República, Montevideo, Uruguay) for providing the tooth germs used in this study and greatly appreciate the support of Prof. Nelly Molina-Frechero (Health Care Department, Universidad Autónoma MetropolitanaXochimilco, Mexico City, Mexico) for assistance with statistical methodology.

This work was supported by the Brazilian Federal Agency for the Support and Evaluation of Graduate Education (CAPES) and State of São Paulo Research Foundation (FAPESP).

\section{Conflict of Interest Statement}

The author(s) declared no potential conflicts of interest with respect to the research, authorship, and/or publication of this article.

\section{REFERENCES}

1. Bologna-Molina R, Mosqueda-Taylor A, Lopez-Corella E, et al. Syndecan-1 (CD138) and Ki-67 expression in different subtypes of ameloblastomas. Oral Oncol 2008; 44: 805-11.

2. Guzmán-Medrano $R$, Arreola-Rosales $R L$, Shibayama $M$, Silva-Olivares DA, Bologna-Molina R, Rodríguez MA. Tumor-associated macrophages and angiogenesis: 
a statistical correlation that could reflect a critical relationship in ameloblastoma. Pathol Res Pract 2012; 208: 672-6.

3. Martínez Martínez M, Mosqueda-Taylor A, Carlos R, Delgado-Azañero W, de Almeida OP. Malignant odontogenic tumors: a multicentric Latin American study of 25 cases. Oral Dis 2014; 20: 380-5.

4. Kumamoto $\mathrm{H}$, Ohki K, Ooya K. Association between vascular endothelial growth factor (VEGF) expression and tumor angiogenesis in ameloblastomas. J Oral Pathol Med 2002; 31: 28-34.

5. Chien CY, Su CY, Hwang CF, Chuang HC, Chen CM, Huang CC. High expressions of CD105 and VEGF in early oral cancer predict potential cervical metastasis. J Surg Oncol 2006; 94: 413-7.

6. Sawa $\mathrm{Y}$, Iwasawa $\mathrm{K}$, Ishikawa $\mathrm{H}$. Expression of podoplanin in the mouse tooth germ and apical bud cells. Acta Histochem Cytochem 2008; 41: 121-6.

7. Imaizumi $Y$, Amano I, Tsuruga $E$, et al. Immunohistochemical examination for the distribution of podoplanin-expressing cells in developing mouse molar tooth germs. Acta Histochem Cytochem 2010; 43: 115-21.

8. Raica M, Cimpean AM, Ribatti D. The role of podoplanin in tumor progression and metastasis. Anticancer Res 2008; 28: 2997-3006.

9. Barnes L, Eveson JW, Reihcart P, Sidransky D (eds). WHO international histological classification of tumours, Vol. 9. IARC Press, 2005.

10. Yuan P, Temam S, El-Naggar A, et al. Overexpression of podoplanin in oral cancer and its association with poor clinical outcome. Cancer 2006; 107: 563-9.

11. Weidner N. Intratumor microvessel density as a prognostic factor in cancer. Am J Pathol 1995; 147: 9-19.

12. Vameşu S. Angiogenesis and ER/PR status in primary breast cancer patients: an analysis of 158 needle core biopsies. Rom J Morphol Embryol 2007; 48: 25-31. 
13. Costa AF, Tasso MG, Mariano FV, et al. Levels and patterns of expression of hypoxia-inducible factor-1 $\alpha$, vascular endothelial growth factor, glucose transporter-1 and CD105 in adenoid cystic carcinomas with high-grade transformation. Histopathology 2012; 60: 816-25.

14. Zustin J, Scheuer HA, Friedrich RE. Podoplanin expression in human tooth germ tissues and cystic odontogenic lesions: an immunohistochemical study. J Oral Pathol Med 2010; 39: 115-20.

15. González-Alva P, Tanaka A, Oku Y, et al. Enhanced expression of podoplanin in ameloblastomas. J Oral Pathol Med 2010; 39: 103-9.

16. Okamoto E, Kikuchi K, Miyazaki Y, et al. Significance of podoplanin expression in keratocystic odontogenic tumor. J Oral Pathol Med 2010; 39: 110-4.

17. Wicki A, Christofori $\mathrm{G}$. The potential role of podoplanin in tumour invasion. $\mathrm{Br} \mathrm{J}$ Cancer 2007; 96: 1-5.

18. Tjioe KC, Oliveira DT, Soares CT, Lauris JR, Damante JH. Is podoplanin expression associated with the proliferative activity of ameloblastomas? Oral Dis 2012; 18: 673-9.

19. Sousa-Neto ES, Cangussu MC, Gurgel CA, et al. Interaction of stromal and microvascular components in keratocystic odontogenic tumors. J Oral Pathol Med. 2016 Sep;45(8):557-64.

20. Watanabe S, Kato M, Kotani I, Ryoke K, Hayashi K. Lymphatic Vessel Density and Vascular Endothelial Growth Factor Expression in Squamous Cell Carcinomas of Lip and Oral Cavity: A Clinicopathological Analysis with Immunohistochemistry Using Antibodies to D2-40, VEGF-C and VEGF-D. Yonago Acta Med. 2013 Mar;56(1):29-37.

21. Kumar P, Wang JM, Bernabeu C. CD 105 and angiogenesis. J Pathol 1996; 178: 363-6.

22. Gadbail AR, Hande A, Chaudhary $M$, et al. Tumor angiogenesis in keratocystic odontogenic tumor assessed by using CD-105 antigen. J Oral Pathol Med 2011; 40: 263-9.

This article is protected by copyright. All rights reserved. 
23. Hande AH, Gadbail AR, Sonone AM, Chaudhary MS, Wadhwan V, Nikam A. Comparative analysis of tumour angiogenesis in solid multicystic and unicystic ameloblastoma by using CD 105 (endoglin). Arch Oral Biol 2011; 56: 1635-40.

24. Kumar DV, Hemavathy S, Kulkarni D, Rudraiah PM, Sidramayya Mathpati SK, Priya S. Expression of CD105 in tumor angiogenesis a comparative study (ameloblastoma, keratocystic odontogenic tumor and dentigerous cyst). J Int Oral Health 2015; 7: 23-7.

25. Sánchez-Romero C, Bologna-Molina R, Mosqueda-Taylor A, Paes de Almeida O. Immunohistochemical Expression of GLUT-1 and HIF-1a in Tooth Germ, Ameloblastoma, and Ameloblastic Carcinoma. Int J Surg Pathol 2016; 24: 410-8.

26. Liang X, Yang D, Hu J, Hao X, Gao J, Mao Z. Hypoxia inducible factor-alpha expression correlates with vascular endothelial growth factor- $\mathrm{C}$ expression and lymphangiogenesis/angiogenesis in oral squamous cell carcinoma. Anticancer Res 2008; 28: 1659-66.

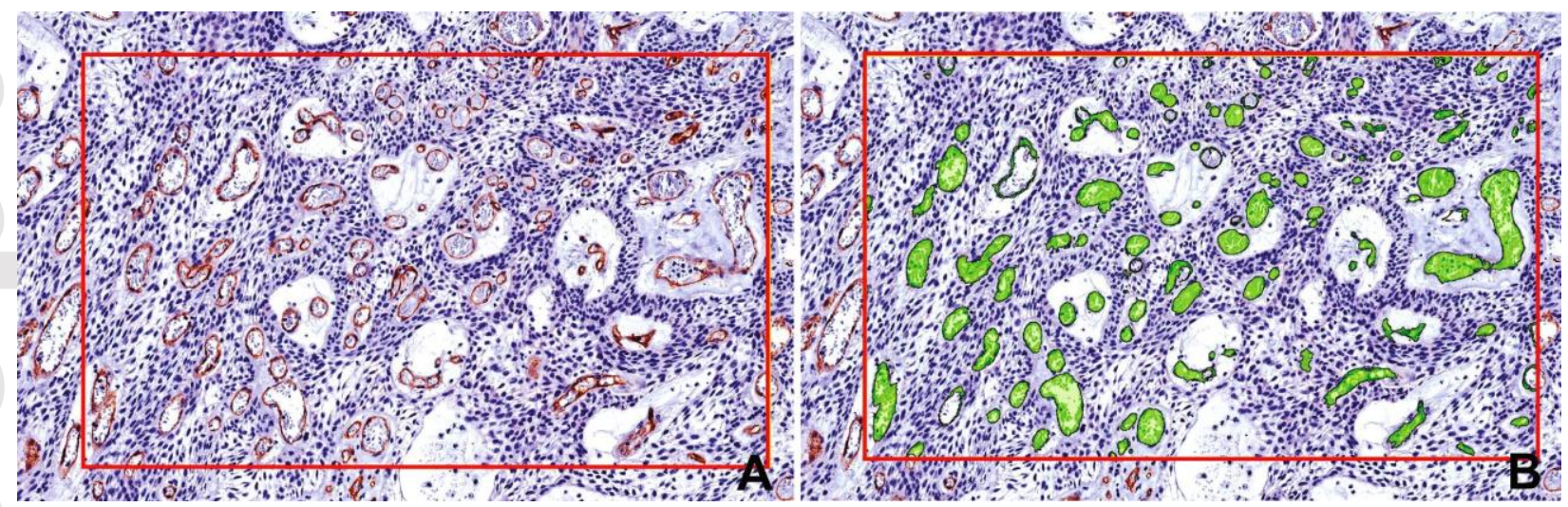

This article is protected by copyright. All rights reserved. 

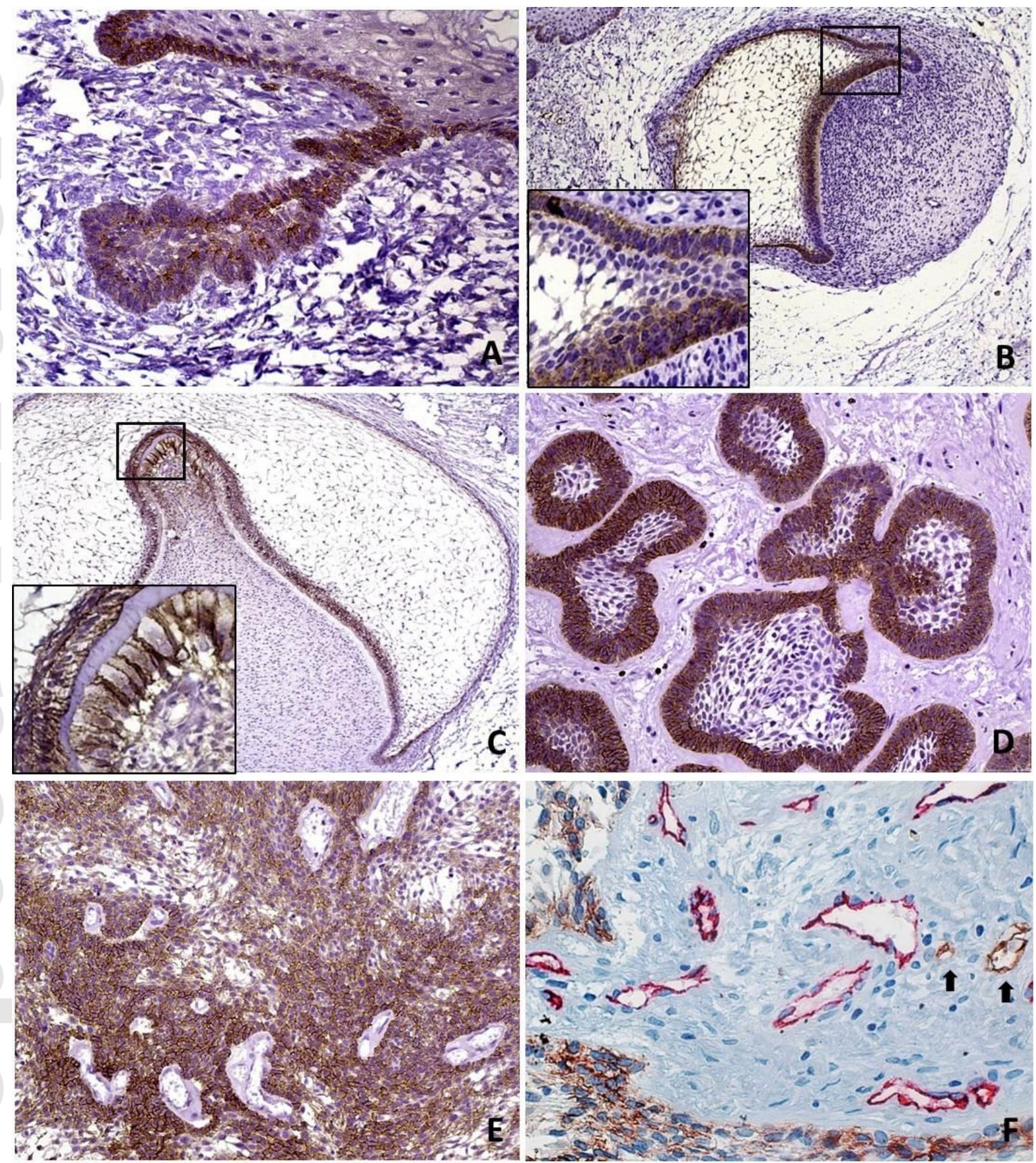

This article is protected by copyright. All rights reserved. 

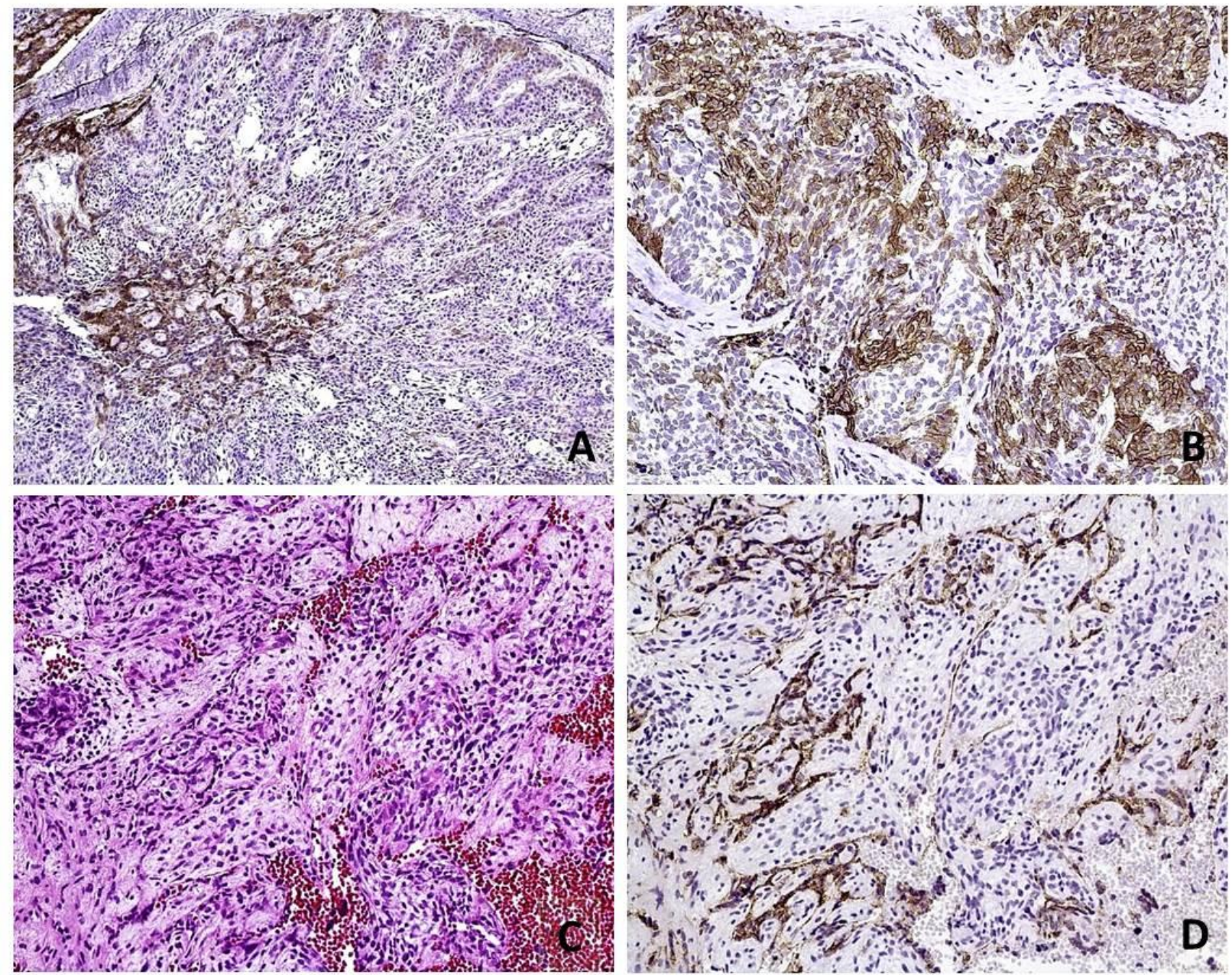

This article is protected by copyright. All rights reserved. 


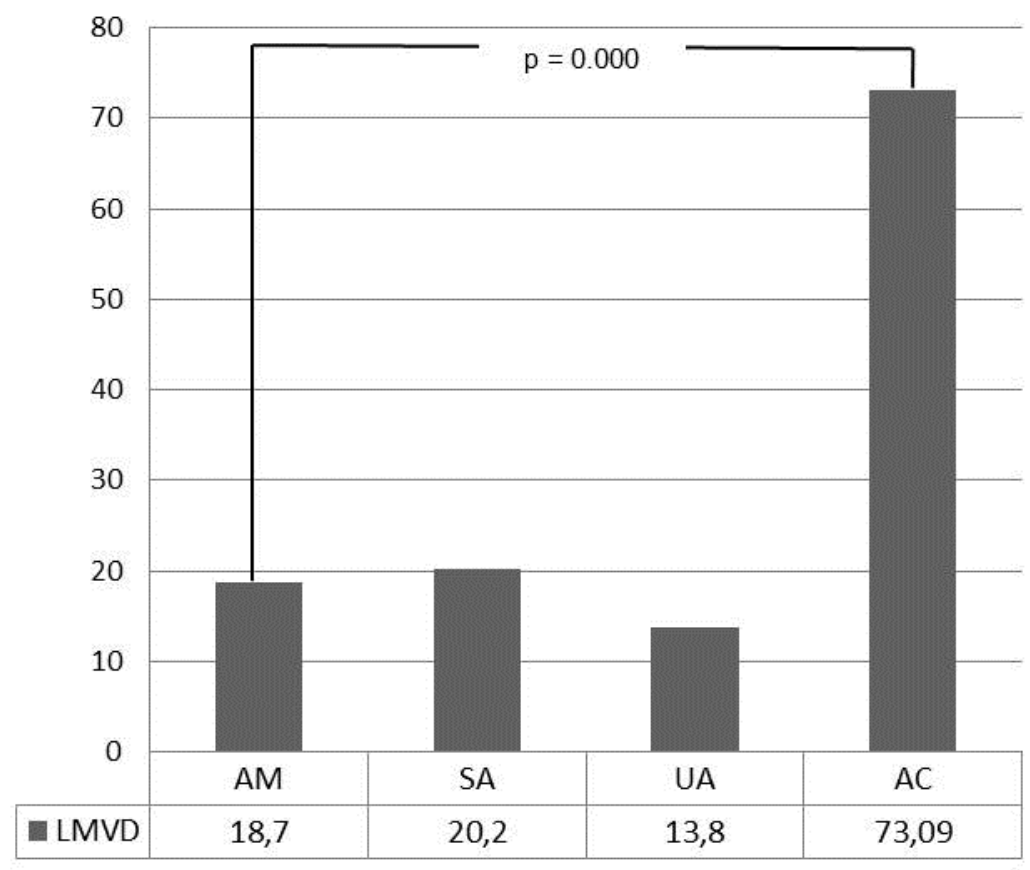

This article is protected by copyright. All rights reserved. 

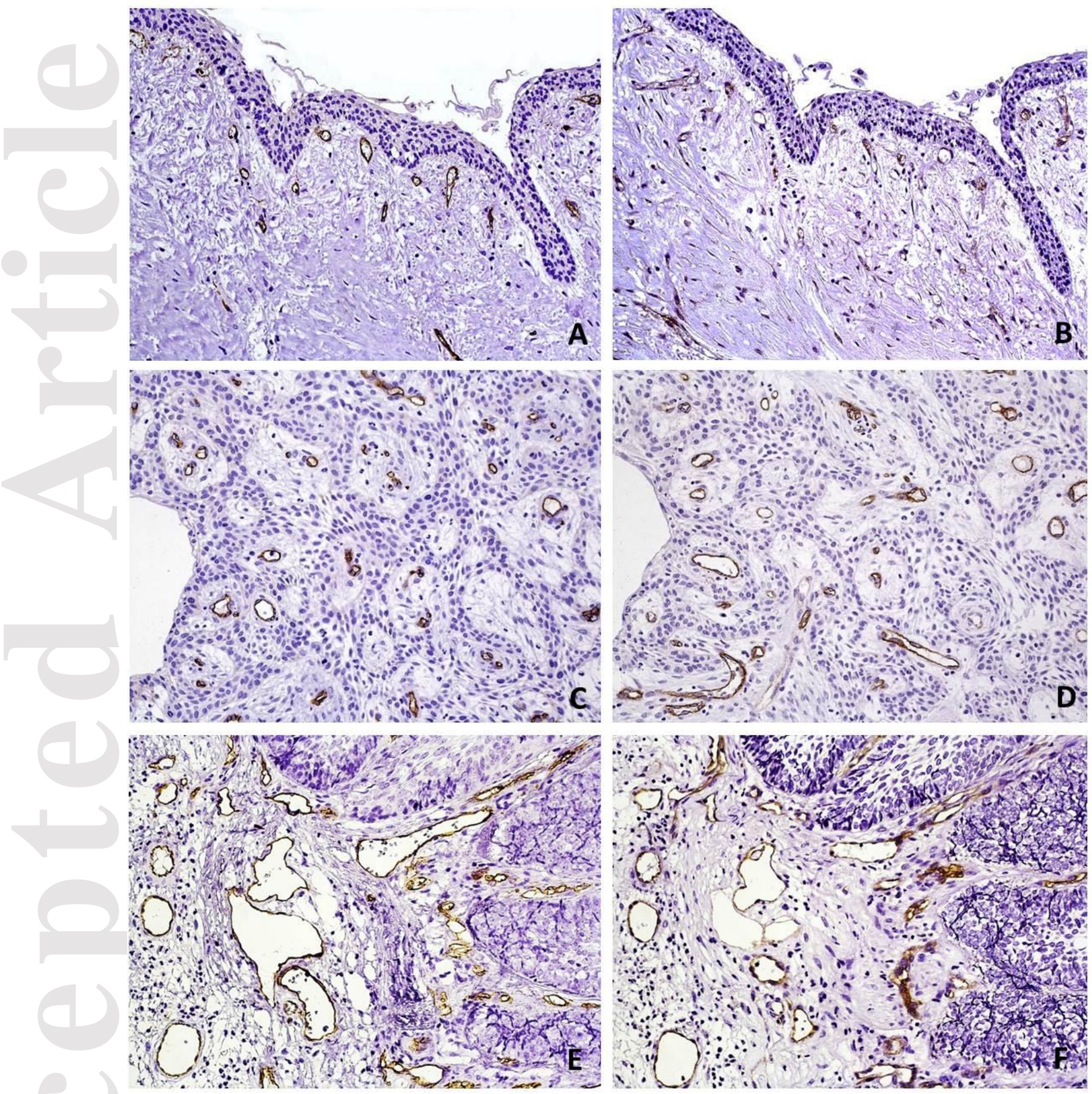

This article is protected by copyright. All rights reserved. 


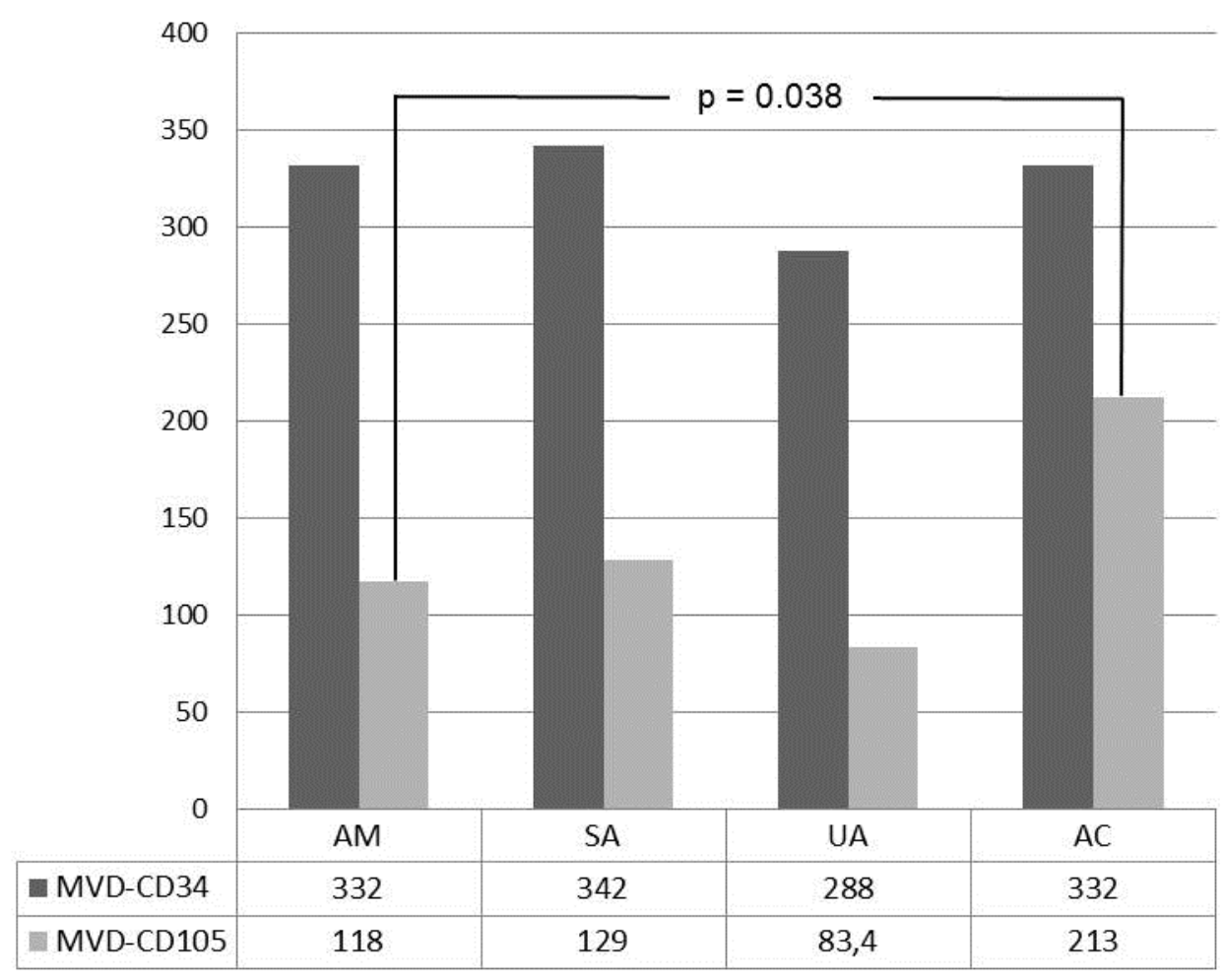

This article is protected by copyright. All rights reserved. 